\title{
Determination of Neutral Lipid and Cluster Formation for Screening Neurocognitive Impairment in Human Immunodeficiency Virus Patients
}

Diego Costaggiu ${ }^{1}$, Luca Serchisu², Claudia Abete ${ }^{1}$, Elisa Pinna ${ }^{1}$, Sarah Vascellari ${ }^{3}$, Francesco Ortu², Paolo Emilio Manconi², Alessandra Pani $^{3}$ and Antonella Mandas ${ }^{1,2^{*}}$

${ }^{1}$ Geriatrics School, University of Cagliari, Italy

${ }^{2}$ Department of Medical Sciences, University of Cagliari, Italy

${ }^{3}$ Department of Biomedical Sciences, University of Cagliari, Italy

\begin{abstract}
Objectives: Despite combination antiretroviral therapy (cART) introduction, human immunodeficiency virus (HIV) patients have increased risk for neurocognitive impairment $(\mathrm{NCl})$. However, $\mathrm{NCl}$ assessment methods in these patients have limitations. Many neurocognitive screening tests, although quicker, identify only the most severe form of impairment and are not suitable for early neurological disorder detection. We previously stated that determination of neutral lipids (NLs) by oil red O (ORO) staining in the cytoplasm of unstimulated peripheral blood mononuclear cells (PBMCs), combined with their tendency to cluster formation (CF), may represent a novel non-invasive approach to detect and monitor neuronal injury in the disease's early stages. In an attempt to find rapid, feasible tools to screen for $\mathrm{NCl}$ in HIV patients, we determined ORO staining and $\mathrm{CF}$ in unstimulated PBMCs from HIV-infected adults.
\end{abstract}

Methods: We analyzed demographic and clinical data including ORO and CF markers in 158 HIV-positive subjects receiving regular HIV infection care.

Results: We found $45 \%$ of HIV patients have higher ORO score (2 to 4 ) and $46 \%$ higher CF score (1-2) compared to age-matched controls. As shown in our previous study, NL accumulation in PBMC cytoplasm appears correlated with cognitive impairment. Also in this HIV patient population, correlation of PBMC NL accumulation with cognitive performance reduction, as highlighted by RBANS, may be indicative of $\mathrm{NCl}$. In addition, according to data given in literature, the prevalence of $\mathrm{NCl}$ in HIV patients is high (about $30-60 \%$ ).

Conclusion: Our data shed light on the fact that as HIV patients have, as indicated by RBANS, lower cognitive performance than controls, and, in addition, that they have higher ORO and CF scores than controls, future studies are needed. Such studies might show that NL/CF screening, together with RBANS, could be useful for the rapid and practical detection of $\mathrm{NCl}$ in HIV-infected patients.

Keywords: HIV; cART; NLs; CF; PBMCs; HAND; Screening for HAND

Abbreviations: ALT: Alanine Aminotransferase; BMI: Body Max Index; cART: Combination Antiretroviral therapy; CEs: Cholesterol Esters; CF: Cluster Formation; CI: Confidence Interval; CNS: Central Nervous System; D-ROMs: Derived Reactive Oxygen Metabolites; HCV+: Hepatitis C Antibody Positivity; HIV: Human Immunodeficiency Virus; MMSE: Mini Mental State Examination; NCI: Neurocognitive Impairment; NLS: Neutral Lipids; ORO: Oil Red O; PBMCs: Peripheral Blood Mononuclear Cells; PBS: Phosphate Buffer Saline; RBANS: Repeatable Battery For The Assessment Of Neuropsychological Status; TIS: Total Index Score of RBANS

\section{Introduction}

Human immunodeficiency virus (HIV) is an infection that weakens the immune system, making it harder for the body to fight infections and disease. It has neurotoxic effects [1], thus it is not surprising that HIV infection can cause problems including neurocognitive impairment (NCI) and dementia [2,3]. Prior to widespread availability of combination antiretroviral therapy (cART), the prevalence of HIVassociated dementia was estimated to be between 6 - 30\% among HIV positive patients [4]. With the advent of cART, the most severe forms of HIV-associated dementia significantly decreased in prevalence, but milder forms of impairment remain highly prevalent and increase with age, affecting about $30-60 \%$ of HIV patients $[5,6]$. It has, however, to be noted that there is current discussion about the prevalence of neurocognitive dysfunction, which might be under- or overestimated, depending on the neurocognitive assessment tools used. In addition, compared to the first decade of the epidemic, a shift has occurred in certain demographic variables and risk factors; for example, increased age and lower transmission among drug users, which might affect the proportion of patients with cognitive impairment [7]. Therefore, understanding what can happen to the central nervous system (CNS) after many years of apparently controlled HIV represents an urgent and important challenge in the field of HIV medicine [8].

Standard comprehensive neuropsychological test batteries exist for the assessment of NCI in HIV patients [9-11]. However, more extensive neuropsychological test batteries, although very useful in highlighting neurocognitive impairment, are difficult to apply in every clinical setting, because they require neuropsychological and neurological expertise and are influenced by differences in education,

*Corresponding author: Antonella Mandas, Department of Medical Sciences University of Cagliari, SS554 bivio Sestu, 09042 Monserrato (CA), Italy; E-mail: amandas@medicina.unica.it

Received September 23, 2015; Accepted October 31, 2015; Published November 05, 2015

Citation: Costaggiu D, Serchisu L, Abete C, Pinna E, Vascellari S, et al. (2015) Determination of Neutral Lipid and Cluster Formation for Screening Neurocognitive Impairment in Human Immunodeficiency Virus Patients. J AIDS Clin Res 6: 517 doi:10.4172/2155-6113.1000517

Copyright: (c) 2015 Costaggiu D, et al. This is an open-access article distributed under the terms of the Creative Commons Attribution License, which permits unrestricted use, distribution, and reproduction in any medium, provided the original author and source are credited. 
culture, and language across borders, and there is a lack of appropriate normative data for many patient populations [12]. Thus, even briefer neurocognitive assessment is required - such as neurocognitive screening instruments - which can be of assistance in the preliminary identification of individuals who might be appropriate for undergoing further testing [12]. However, most common traditional screening tools have wide limitations, are often underpowered, and attempt to identify only the most severe form of impairment. MMSE, for example - which, thanks to its rapidity of execution and ease of interpretation, is in the diagnostic toolbox of most clinicians - unfortunately has poor sensitivity and specificity for NCI in HIV infection, being more useful for the identification of cortical dementia syndromes (such as Alzheimer's disease) and is lacking when it comes to investigating cognitive domains of attention and working memory, typically damaged in NCI during HIV infection [13]. On the other hand, help can be obtained through the use of a surrogate marker, like neutral lipid accumulation (NLs) in cytoplasm of unstimulated peripheral blood mononuclear cells (PBMCs). In fact, we have recently reported that accumulation of NLs, mainly cholesterol esters (CEs), occur in PBMCs in many different diseases characterized by the deterioration of cognitive functions, as well as in subjects with symptomatic pre-dementia, but who preserve the ability to function independently in daily life, which is referred to as mild cognitive impairment [14,15]. Interestingly, NL content in PBMCs was significantly correlated with clinical cognitive severity. Among methods utilized, staining of NLs with oil red O (ORO), a lipid-soluble bright red dye, was the cheapest, fastest, and easiest to perform, and was one that required less material. Therefore, we propose determination of NLs in PBMCs by ORO staining, as a useful tool for early detection of $\mathrm{NCI}$ in clinical practice.

Another interesting finding of our studies was that freshly isolated, namely unstimulated, PBMCs, from subjects with and/or at risk of cognitive impairment merge together to form aggregates or clusters more or less visible under light microscope. The mechanism by which PBMCs form the clusters remains to be determined. However, since clusters observed resemble those seen in growth-stimulated PBMCs, we assumed that cluster formation (CF), may reflect a preexisting in vivo activated state of PBMCs, probably secondary to the release in plasma, by a damaged brain, of inflammatory mediators such as cytokines $[16,17]$. We suggest that the presence of NLs in the cytoplasm of unstimulated-PBMCs, combined with their potential tendency to form clusters, may represent a non-invasive novel approach to detect and monitor neuronal injury in the early stages of the disease.

The aim of this work was to find brief and feasible tools to screen for NCI in HIV patients. In order to do this we determined ORO staining and CF in unstimulated PBMCs from patients affected by HIV infection.

\section{Methods}

\section{Study population}

We studied 158 Caucasian HIV-infected patients (mean age 42.1 \pm 9.2 years; 83 men and 75 women) enrolled at the outpatient services of infectious diseases at the University Hospital of Cagliari. Exclusion criteria included severe premorbid or comorbid psychiatric disorders, confounding neurological disorders such as chronic seizures, stroke, cranial trauma resulting in loss of consciousness of more than 30 minutes, multiple sclerosis, brain tumors. Informed consent was obtained from all participants prior to enrollment. The University of Cagliari Review Board approved the study.
As control group (Control 1), we used results from 112 previously evaluated, apparently healthy Caucasian subjects, mean age $44.8 \pm 9.5$, of whom 30 females and 82 males, with no personal or family history of neurological or psychiatric disorders. They underwent blood sampling for oxidative balance assessment and PBMC neutral lipid determination. The RBANS results obtained in HIV patients were compared with those obtained in the healthy Italian population used for test validation in the Italian version (Control 2) [18].

\section{Measures}

Measures included: age, sex, CD4+ counts, duration of known infection from the diagnosis, years on cART, HIV RNA viral load, hepatitis $\mathrm{C}$ antibody positivity ( $\mathrm{HCV}+$ ), OXY-adsorbent (OXY) and derivative reactive oxygen metabolites (d-ROMs) tests, glycemia, alanine aminotransferase (ALT), cholesterolemia, trygliceridemia, body max index (BMI), mini mental state examination (MMSE) and repeatable battery for the assessment of neuropsychological status (RBANS) total score.

Levels of plasma total cholesterol, triglycerides, glycemia and ALT were determined using standard enzymatic methods. HIV-RNA viral load is expressed as copies $/ \mathrm{mL}$.

\section{d-ROMs test}

Serum total oxidant capacity was determined by performing the d-ROMs test [19]. The d-ROMs test permits the measurement in the blood of alkoxyl (RO) and peroxyl (ROO) radicals that are produced in the presence of iron (which is released from plasma proteins by anacidic buffer), according to Fenton's reaction. Such radicals, in turn, are able to oxidize an alkyl-substituted aromaticammine (A-NH2, that is dissolved in chromogenic mixture) thus transforming them into a pink-colored derivative. Finally, this colored-derivative is photometrically quantified. Indeed, the intensity of developed color is directly proportional to the level of ROMs, according to Lambert-Beer's law. The results of the d-ROMs test were expressed as Carratelli Units (CARR U). Blood ROMs level (mainly hydroperoxides) normal values in a population of healthy subjects, as measured by d-ROMs test, have a unimodal distribution that peaks between 250 and 300 CARR U.

\section{OXY-Adsorbent test}

The OXY-Adsorbent test evaluates the plasma/serum ability to oppose the massive oxidative action of a hypochlorous acid ( $\mathrm{HClO}$ ) solution [19]. Briefly, in the OXY-Adsorbent test the efficacy of the blood antioxidant system is monitored indirectly by measuring the excess of $\mathrm{HClO}$ in serum (released as a radical). As unreacted radicals of $\mathrm{HClO}$ react with a correctly buffered chromogenic substrate $(\mathrm{N}, \mathrm{N}$ diethylparaphenylendiamine), a colored complex develops that can be measured photometrically, presenting a maximum peak of adsorbance at 505 or $546 \mathrm{~nm}$. The concentration of the colored complex is directly proportional to the concentration of $\mathrm{HClO}$ and indirectly related to antioxidant capacity. In healthy subjects, the OXY-Adsorbent Test has a value exceeding $350 \mathrm{mmol} / \mathrm{L}$. In normal practice $1 \mathrm{~mL}$ of plasma is able to "adsorb" 350 micromoles of hypochlorous acid. Values below $350 \mathrm{mmol} / \mathrm{L}$ are indicative of an oxidative stress condition because of a lowering of antioxidant defenses.

\section{RBANS}

RBANS is a screening instrument used to evaluate general cognitive function [20]. The RBANS is composed of 12 subtests that yield 5 index scores and a total score. The subtests of list learning and story memory comprise the immediate memory index; figure copy and line 
orientation yield the visuospatial/constructional index; picture naming and semantic fluency yield the language index; and digit span and digit symbol test comprise the attention index. Four subtests make up the delayed memory index, specifically, list recall, list recognition, story recall, and figure recall. All the index scores are summed to calculate a total index score (TIS). Normative information, which is used to calculate the indexes and TIS, is based on an Italian sample of 356 healthy adults who range in age from 20 to 79 years [18]. Published normative data tables were used to produce control data, which were individually matched to the test group for age.

\section{MMSE}

MMSE test, corrected for age and education, is used for evaluating cognitive status. Thirty correct-answer points indicate cognitive deficit absence, and 0 , maximum cognitive deficit. A score of less than 24 out of 30 points is suggestive of cognitive deficit [21.22].

\section{ORO staining and cell clustering}

NLs in PBMCs were analyzed by ORO staining method [16]. In detail, a small amount of whole blood was collected into commercially available anticoagulant-treated tubes. For the isolation of PBMCs, $500 \mu \mathrm{l}$ of Ficoll were placed into a $1.5 \mathrm{~mL}$ Eppendorf tube with an equal amount of anticoagulant. The tubes were then centrifuged in a centrifuge-like Eppendorf at 5,000 x g for 10 minutes. After centrifugation the white ring containing the PBMCs was taken out with the help of micropipettes and carefully placed in 6-well tissue culture plates. PBMCs were washed three times with phosphate buffered saline (PBS), and fixed in 10\% neutral buffered formalin (10 min). Cells were then treated with isopropyl alcohol (60\%), washed, stained with $1 \mathrm{ml}$ of ORO working solution and incubated at room temperature for a few minutes. Hematoxylin counterstain was used to visualize the nucleus. ORO was then removed and cells washed with PBS 3 times, 3 min each. After ORO staining, cells were imaged using an inverted phase microscope fitted with a digital camera with a magnification of 400 . At least two different fields per sample were imaged and analyzed. The red intensity was scored on a semi-quantitative scale (from 0 to 4 ) by two blinded observers: 0 indicated no staining; 1 , rare positive cells or staining barely visible; 2 , focal staining or faint diffuse staining clearly visible at low power; 3 , multifocal staining or moderate diffuse staining; and 4 , intense diffuse staining. Grades representing the proportion of PBMCs clustered were rated using a semiquantitative scale from 0 to 2 in which 0 indicates single cells or very few aggregated cells, 1 , moderate aggregated cells, 2 , highly aggregated cells.

\section{Statistical analysis}

Quantitative variables were expressed as means \pm standard deviation $(\mathrm{M} \pm \mathrm{SD})$. Variables associated with NCI were described as means and compared using the $t$-test and the Mann-Whitney test. In some experiments we converted variables into a binary version. When appropriate $\chi 2$ tests and Pearson's correlation were also applied. Positive and negative predictive values were also calculated and reported with their $95 \%$ confidence intervals (CI). Excel data analysis and XLSTAT 2014 were employed for all analyses. A $P$ value $<0.05$ was considered statistically significant.

\section{Results}

Characteristics of HIV patients in cART are displayed in Table 1. They include: age, sex, CD4+ counts, duration of known infection from the diagnosis, years on cART, HIV RNA viral load, $\mathrm{HCV}+$, OXY and d-ROMs tests, glycemia, ALT, cholesterolemia, trygliceridemia, BMI,
MMSE and RBANS TIS. According to a previous study, serum oxidant levels (d-ROM-test) were higher in the HIV-treated group [23]. The results of other clinical chemistry parameters were within the expected ranges.

Given that HIV patients are reported to exhibit neuropsychological performance profiles, we also examined the relative outcomes with established neurocognitive screening tools, including MMSE and RBANS TIS. The average MMSE score of the study group was $29.0 \pm 3.3$, which indicates normal cognitive function, while that of RBANS TIS score was $85.1 \pm 13.4$, which is interpreted as one standard deviation below the mean score of age-matched Control 2. Comparisons between normative data curves (Figure 1) generated from age-matched Control 2, and those obtained from HIV patients, clearly indicate that the percentage of RBANS TIS greatly differs between the two groups. In

\begin{tabular}{|c|c|}
\hline Variables & $\begin{array}{l}\text { HIV patients } \\
\text { no. } 158\end{array}$ \\
\hline Age, years $(\mathrm{M} \pm \mathrm{SD})$ & $42.1 \pm 9.25$ \\
\hline Education, years $(\mathrm{M} \pm \mathrm{SD})$ & $10.4 \pm 4.1$ \\
\hline 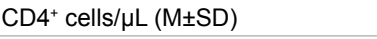 & $611 \pm 313$ \\
\hline Years post-HIV diagnosis $(\mathrm{M} \pm \mathrm{SD})$ & $14.0 \pm 4.6$ \\
\hline Years on cART therapy $(\mathrm{M} \pm \mathrm{SD})$ & $8.4 \pm 3.9$ \\
\hline $\operatorname{Sex}(M / F)$ & $83 / 75$ \\
\hline HIV-RNA (M $\pm S D)$ & $3431 \pm 13696$ \\
\hline $\mathrm{HCV}+($ no.) & 83 \\
\hline 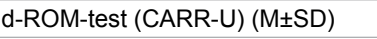 & $364.7 \pm 70.7$ \\
\hline Oxy-test (mmol/L) (M $\pm S D)$ & $395.6 \pm 54.6$ \\
\hline Glycemia (mg/dl) (M $\pm S D)$ & $88.8 \pm 19.3$ \\
\hline ALT (U/ml) (M $\pm S D)$ & $46.7 \pm 41.4$ \\
\hline Cholesterolemia (mg/dl) $(\mathrm{M} \pm \mathrm{SD})$ & $184.2 \pm 38,8$ \\
\hline Tryglicerides (mg/dl) (M $\pm S D)$ & $193.7 \pm 177.3$ \\
\hline $\mathrm{BMI}\left(\mathrm{Kg} / \mathrm{m}^{2}\right)(\mathrm{M} \pm \mathrm{SD})$ & $22.9 \pm 2.6$ \\
\hline MMSE (score) (M $\pm S D)$ & $29.0 \pm 3.3$ \\
\hline RBANS TIS (M $\pm S D)$ & $85.1 \pm 13.4$ \\
\hline
\end{tabular}

Normally distributed parameters are expressed as Mean \pm SD.

Table 1: Characteristics of HIV patients.

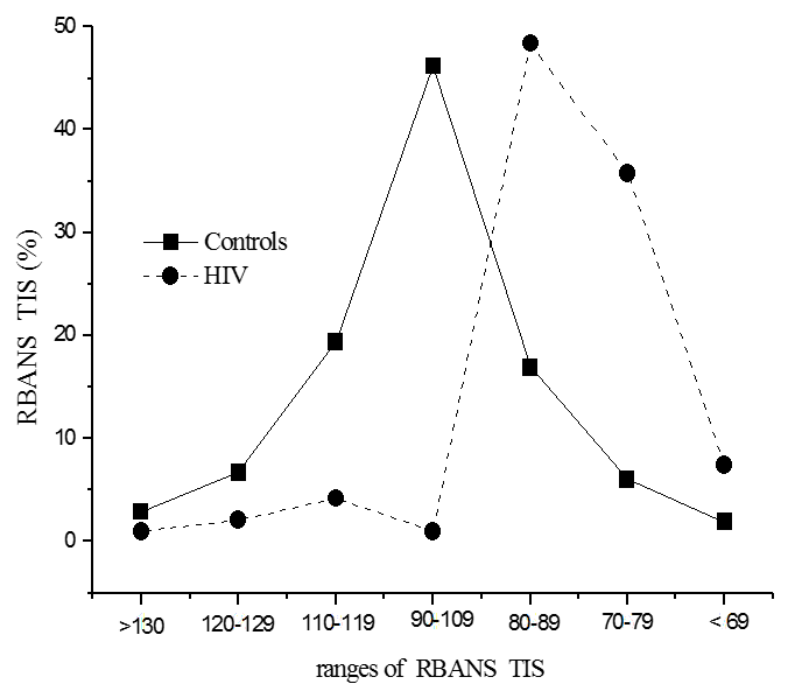

Figure 1 (Legend): Comparisons between normative data curves generated from age-matched controls and those obtained from HIV patients. The figure shows an unimodal distribution of HIV patients scores that greatly differs from that of control subjects, being the mean RBANS TIS of the first group one standard deviation below the mean of age-matched controls. 
particular, while the percentage of scores in controls in the ranges of 90 $109,110-119,120-129$ and $\geq 130$, indicating average, average-to-high, high and very high score, respectively, were $46.2 \%, 19.4 \%, 6.7 \%$ and $2,9 \%$; in HIV patients they were $1 \%, 4.2 \%, 2.1 \%, 1 \%$. In HIV patients the mean score was found to be in the range $80-89$ (48.4\% in HIV vs $16.9 \%$ in Control 2) (Figure 1).

It would appear from these results that RBANS is able to identify a greater proportion of subjects who may be at risk of developing NCI (longitudinal evaluation would be required), while MMSE is unable to distinguish between age-matched healthy subjects and HIV patients, according to previous studies $[13,24,25]$.

Next, we determined CF and ORO scores in unstimulated PBMCs from HIV patients. A significant positive correlation was found between $\mathrm{CF}$ and ORO scores in HIV patients $(\mathrm{r}=0.888, P<0.001)$. As shown in Table 2, mean age was slightly but significantly lower in HIV patients compared to Control 1. The rate of ORO score was much higher in PBMCs from HIV patients compared to Control 1. Grouping ORO scores of 0 and 1 together as "low score" and 2 to 4 as "high score", we found significant differences in the number of subjects with ORO high score between HIV patients and Control 1 (45\% and $8 \%$ respectively). The calculated values of $\chi 2$ between the two groups are reported in Table 3. In HIV patients, importantly, an MMSE score of <24, (which is the limit for impaired cognition) was detected in 8 subjects (5\%), all of which had ORO high score. We also found that freshly isolated (unstimulated) PBMCs from HIV patients tend to aggregate in vitro in small-to-medium clusters, hence resembling cultured PBMCs after

\begin{tabular}{|l|c|c|c|c|}
\hline Variables & $\begin{array}{c}\text { Controls } \\
\text { no. 112 }\end{array}$ & $\begin{array}{c}\text { HIV } \\
\text { no. 158 }\end{array}$ & $\boldsymbol{P}$ value & $\begin{array}{c}\mathbf{X}^{\mathbf{2}}(\boldsymbol{P}) \\
\text { OR (Cl) }\end{array}$ \\
\hline Age (M \pm SD) & $44.8 \pm 9.5$ & $42.1 \pm 9.2$ & 0.020 & \\
\hline ORO score & $0.5 \pm 0.7$ & $1.6 \pm 1.24$ & 0.000 & \\
\hline ORO score (0-1) & no. 102 & no. 87 & & $40.5(0.0000)$ \\
\hline ORO score (2-4) & no. $10(8.9 \%)$ & no. $71(44.9 \%)$ & & \\
\hline CF score & $0.35 \pm 0.24$ & $0.8 \pm 1.06$ & 0.000 & \\
\hline CF score (0) & no. 100 & no. 86 & & $37.2(0.0000)$ \\
\hline CF score (1-2) & no. $12(10.7 \%)$ & no. $72(45.6 \%)$ & & \\
\hline
\end{tabular}

Table 2: Comparison of ORO staining and CF between HIV patients and agematched controls.

\begin{tabular}{|l|c|c|c|}
\hline Variables & $\begin{array}{c}\text { ORO-L } \\
\text { no. 86 }\end{array}$ & $\begin{array}{c}\text { ORO H } \\
\text { no. 72 }\end{array}$ & $\begin{array}{c}\text { Chi square (P) } \\
\text { OR (IC) }\end{array}$ \\
\hline CF-L & 49 & 37 & $0.5(0.4824)$ \\
CF-H & 37 & 35 & $1.3(0.7-2.4)$ \\
\hline Age-L & 45 & 39 & $0.1(0.8173)$ \\
\hline Age-H & 41 & 33 & $0.9(0.5-1.7)$ \\
\hline F & 40 & 36 & $0.2(0.6621)$ \\
M & 46 & 36 & $0.9(0.5-1.6)$ \\
\hline ROM-L & 26 & 20 & $0.1(0.7352)$ \\
ROM H & 60 & 52 & $1.13(0.6-2.3)$ \\
\hline OXY-L & 67 & 57 & $0.0(0.8478)$ \\
\hline OXY H & 19 & 15 & $0.9(0.4-2.0)$ \\
\hline CD4-<200 & 38 & 30 & $0.1(0.7501)$ \\
\hline CD4-> 200 & 48 & 42 & $0.9(1.5-1.7)$ \\
\hline HVR-L & 57 & 47 & $0.0(0.8949)$ \\
\hline HVR-H & 29 & 25 & $1.1(0.5-2.0)$ \\
\hline Smoke no & 34 & 44 & $0.0(0.9340)$ \\
\hline Smoke yes & 52 & 34 & $1.0(0.5-2)$ \\
\hline HCV neg & 40 & 38 & $0.0(0.9290)$ \\
\hline HCV pos & 46 & & $1.0(0.5-1.8)$ \\
\hline
\end{tabular}

Normally distributed parameters are expressed as dichotomous variables Table 3: Association between ORO low or high score and variables considered. mitogen activation with phytohemagglutinin [14,15]. By rating CF using a semiquantitative scale from 0 to 2 , we found that CF follows a pattern similar to ORO staining, with CF score being significantly higher in HIV patients compared to Control 1. When CF score was classified as dichotomous variables (low $=0$ ) and (high $=1-2$ ), we found that the percentage of subjects with high CF was $46 \%$ for HIV patients and $11 \%$ for Control 1 .

By converting variables into a binary version, comparison of ORO staining and CF between HIV patients and age-matched Control 1 showed that none of the clinical variables considered were associated with ORO or CF scores (table 3).

To verify whether NLs accumulation and cognitive performance, measured with RBANS, were affected by viral load (VL), we performed further correlation analysis. In particular, we carried out, on 158 HIV patients, a Pearson's correlation between VL and, respectively, ORO score, CF score and RBANS TIS. This correlation appeared not significant $(r=0.153, P=0.139 ; r=0.127, P=0.219 ; r=-0.450, P=0.665$, respectively). Excluding patients with high HIV VL, so performing the same correlation analysis only including $130 \mathrm{HIV}$ patients with VL $<500$ copies $/ \mathrm{mL}$, we obtained the same results $(r=0.003, P=0.982 ; r=$ $0.176, P=0.879 ; r=-0.067 P=0.563$, respectively), in agreement with literature, which indicates that viral suppression on treatment is not sufficient to avoid development or persistence of NCI [2].

Finally we performed Pearson's correlation between the NL parameters (ORO and CF score) and the scores obtained by MMSE and RBANS TIS from HIV patients. We found a significant negative correlation both between ORO score and RBANS TIS and between CF score and RBANS TIS ( $r=-0.406, P<0.001 ; r=-0.380, P<0.001$ respectively), while the Pearson's correlation between ORO and MMSE scores and between CF and MMSE scores were not significant $(\mathrm{r}=$ $-0.119, P=0.249 ; \mathrm{r}=-0.068, P=0.513$, respectively).

\section{Discussion}

This study collected and examined data from a sample of 158 HIV patients who were referred for assessment of neurocognitive function. The aim of this work was to find a brief and feasible method for early NCI diagnosis in HIV patients, particularly to detect those milder forms - currently the most widespread and at the same time, most difficult to detect without sensitive enough instruments. In order to do this we evaluated the presence of NLs by ORO staining in the cytoplasm of unstimulated-PBMCs combined with their potential tendency to form clusters, which we have previously proposed as noninvasive novel approaches to detect and monitor neuronal injury in the early stages of the disease $[14,15]$. For comparison, in the same patients we examined MMSE and RBANS, which are among the common procedures used for assessment of neurocognitive function. Evaluation of MMSE mean scores showed that the HIV patient group achieved scores generally within the normal range, indicating that MMSE is not a good choice for early detection of NCI in HIV patients, according to previous studies $[13,24,25]$. RBANS demonstrates better efficiencies in discriminating between controls and HIV patients, but, unfortunately, it is made complicated by the necessity of patients being available. Furthermore, the limit of this test is the impossibility of obtaining individual standardized scores for each subtest administered, because of the fact that they are grouped by cognitive domain [20]

By measuring ORO and CF scores in PBMCs we found that HIV patients have significantly higher ORO and CF scores compared to Control 1. The percentage of subjects having high ORO scores (2 to 4) was $45 \%$ for HIV patients and $8.9 \%$ for Control 1, while that of high 
CF (1 to 2 ) was $46 \%$ for HIV and $10.7 \%$ for Control 1 . These results, as in previous studies reporting an elevated prevalence of neurocognitive dysfunction (about 30-60\% of HIV patients) [5,6], may indicate a high frequency of NCI in our sample of HIV patients, which mainly comprised middle-aged men on cART with a heterogeneous spectrum of clinical conditions.

The significant negative correlations both between ORO score and RBANS TIS and between CF score and RBANS TIS seem to strengthen this hypothesis. However, the same correlations were not seen between ORO and MMSE scores nor between CF and MMSE scores: this could be due to the small number of patients with MMSE score $<24$, in accordance with literature data indicating that MMSE is not useful for early NCI diagnosis in HIV infection [13,24,25].

Other tools have been proposed as screening methods for NCI in HIV patients. One of the most widely studied is the HIV Dementia Scale [24]. Nevertheless, this scale was designed to screen for dementia, which is considerably less prevalent nowadays thanks to cART [2]. Consequently, some authors have warned about the limitations of using the HIV Dementia Scale to screen for milder forms of NCI, highlighting that their main application should be to screen for dementia or severe forms of impairment [24-29]. Our priority was to find brief and feasible tools to screen for early detection of NCI in HIV patients that could be easily applied in clinical practice. Therefore, we placed emphasis on establishing whether NL determination by ORO staining in unstimulated PBMCs, combined with their potential tendency to form clusters, may represent a non-invasive novel approach to detect and monitor neuronal injury also in HIV patients. The potential advantages of ORO/CF method are its great ease of application, its cost, and the small quantity of blood required. Furthermore, this screening could integrate a brief neuropsychological assessment battery in longitudinal clinical follow-up.

However, the results obtained from this study, although interesting, are insufficient to assert that NL accumulation in unstimulated PBMCs, determined by ORO staining and $\mathrm{CF}$, is definitely correlated to NCI in HIV infection. Therefore, although our data shed light on the fact that HIV patients have, with RBANS, lower performance than controls, and, in addition, that they have higher ORO and CF scores than controls, future studies are needed. These studies should include, both in HIV patients and the control group, cognitive assessment by RBANS and NL determination in unstimulated PBMCs by ORO staining. Moreover, the diagnostic validity of RBANS for detecting NCI in HIV infection should be tested through direct comparison with an extensive neuropsychological battery (which contains previously validated tests capable of evaluating cognitive domains typically involved in NCI during HIV infection) and, above all, through longitudinal monitoring of HIV patients.

\section{Acknowledgements}

This paper is dedicated to the fond memory of Prof. Sandra Dessì, with whom we collaborated and on whom we could always count for many years.

\section{References}

1. Valcour V, Sithinamsuwan P, Letendre S, Ances B (2011) Pathogenesis of HIV in the central nervous system. Curr HIVIAIDS Rep 8: 54-61.

2. Heaton RK, Franklin DR, Ellis RJ, McCutchan JA, Letendre SL, et al. (2011) HIV-associated neurocognitive disorders before and during the era of combination antiretroviral therapy: differences in rates, nature, and predictors. J Neurovirol 17: 3-16

3. Simioni S, Cavassini M, Annoni JM, Rimbault Abraham A, Bourquin I, et al (2010) Cognitive dysfunction in HIV patients despite long-standing suppression of viremia. AIDS 24: 1243-1250.
4. Heaton RK, Clifford DB, Franklin DR Jr., Woods SP, Ake C, et al. (2010) HIVassociated neurocognitive disorders persist in the era of potent antiretroviral therapy: CHARTER Study. Neurology 75: 2087-2096.

5. Heaton RK, Grant I, Butters N, White DA, Kirson D, et al. (1995) The HNRC 500--neuropsychology of HIV infection at different disease stages. HIV Neurobehavioral Research Center. J Int Neuropsychol Soc 1: 231-251.

6. Grant I (2008) Neurocognitive disturbances in HIV. Int Rev Psychiatry 20: 3347.

7. Heaton RK, Marcotte TD, Mindt MR, Sadek J, Moore DJ, et al. (2004) The impact of HIV-associated neuropsychological impairment on everyday functioning. J Int Neuropsychol Soc 10: 317-331.

8. Schouten J, Cinque P, Gisslen M, Reiss P, Portegies P (2011) HIV-1 infection and cognitive impairment in the cART era: a review. AIDS 25: 561-575.

9. Antinori A, Arendt G, Becker JT, Brew BJ, Byrd DA, et al. (2007) Updated research nosology for HIV-associated neurocognitive disorders. Neurology 69: 1789-1799.

10. Muñoz-Moreno JA (2007) Neurocognitive and motor disorders in HIV infection. Assessment and interventions. In: Plishe VN, (ed.) Research Focus on Cognitive Disorders. Hauppauge, NY: Nova Science Publishers, Inc; pp. 133147.

11. Mind Exchange Working Group (2013) Assessment, diagnosis, and treatment of HIV-associated neurocognitive disorder: a consensus report of the mind exchange program. Clin Infect Dis 56: 1004-1017.

12. Robertson K, Liner J, Heaton R (2009) Neuropsychological assessment of HIVinfected populations in international settings. Neuropsychol Rev 19: 232-249.

13. Valcour VG (2011) Evaluating cognitive impairment in the clinical setting practical screening and assessment tools. Top Antivir Med 19: 175-180.

14. Pani A, Mandas A, Diaz G, Abete C, Cocco PL, et al. (2009) Accumulation of neutral lipids in peripheral blood mononuclear cells as a distinctive trait of Alzheimer patients and asymptomatic subjects at risk of disease. BMC Med 7: 66.

15. Mandas A, Abete C, Putzu PF, La Colla P, Dessì S, et al. (2012) Changes in cholesterol metabolism-related gene expression in peripheral blood mononuclear cells from Alzheimer patients. Lipids Health Dis 11: 39.

16. Mandas A, Dessì S (2014) Mononuclear cells in dementia. Clin Chim Acta 431C: $278-287$.

17. Peiretti E, Mandas A, Abete C, Vinci M, Piludu S, et al. (2014) Age-related macular degeneration and cognitive impairment show similarities in changes of neutral lipids in peripheral blood mononuclear cells. Exp Eye Res 124: 11-16.

18. Ponteri M, Pioli R, Padovani A, Tunesi S, De Girolamo G (2007) RBANS Repeatable Battery for the Assessment of Neuropsychological Status. (Italian adaptation), Giunti OS.

19. Vassalle $C$ (2008) An easy and reliable automated method to estimate oxidative stress in the clinical setting. Methods Mol Biol 477: 31-39.

20. Randolph C, Tierney MC, Mohr E, Chase TN (1998) The Repeatable Battery for the Assessment of Neuropsychological Status (RBANS): preliminary clinical validity. J Clin Exp Neuropsychol 20: 310-319.

21. Folstein MF, Folstein, SE, McHugh PR (1975) "Mini-Mental state". A practica method for grading the cognitive state of patients for the clinician. J Psychiatr Res 12: 189-198.

22. Measso G, Cavarzeran F, Zappalà G, Lebowitz, BD, Crook TH, et al. (1993) The Mini-Mental State Examination: normative study of a random sample of Italian population. Dev Neuropsychol 9: 77-85.

23. Mandas A, lorio EL, Congiu MG, Balestrieri C, Mereu A, et al. (2009) Oxidative imbalance in HIV-1 infected patients treated with antiretroviral therapy. J Biomed Biotechnol 2009: 749575 .

24. Power C, Selnes OA, Grim JA, McArthur JC (1995) HIV Dementia Scale: a rapid screening test. J Acquir Immune Defic Syndr Hum Retrovirol 8: 273-278.

25. McArthur JC, Brew BJ (2010) HIV-associated neurocognitive disorders: is there a hidden epidemic? AIDS 24: 1367-1370.

26. Smith CA, van Gorp WG, Ryan ER, Ferrando SJ, Rabkin J (2003) Screening subtle HIV-related cognitive dysfunction: the clinical utility of the HIV dementia scale. J Acquir Immune Defic Syndr 33: 116-118. 
Citation: Costaggiu D, Serchisu L, Abete C, Pinna E, Vascellari S, et al. (2015) Determination of Neutral Lipid and Cluster Formation for Screening Neurocognitive Impairment in Human Immunodeficiency Virus Patients. J AIDS Clin Res 6: 517. doi:10.4172/2155-6113.1000517

Page 6 of 6

27. Bottiggi KA, Chang JJ, Schmitt FA, Avison MJ, Mootoor Y, et al. (2007) The HIV Dementia Scale: predictive power in mild dementia and HAART. J Neurol Sci 260: 11-15.

28. Waldrop-Valverde D, Nehra R, Sharma S, Malik A, Jones D, et al. (2010)
Education effects on the International HIV Dementia Scale. J Neurovirol 16: 264-267.

29. Sakamoto M, Marcotte TD, Umlauf A, Franklin D Jr, Heaton RK, et al (2013) Concurrent classification accuracy of the HIV dementia scale for HIVassociated neurocognitive disorders in the CHARTER cohort. J Acquir Immune Defic Syndr 62: 36-42. 